\title{
Limiting Internal Supply Voltage Spikes in DC-DC Converters
}

\author{
José Rocha ${ }^{(1)}$ \\ Instituto Superior de \\ Engenharia de Lisboa / \\ IPL $^{(1)}$, Lisboa, Portugal \\ jrocha@deetc.isel.ipl.pt
}

\author{
Marcelino Santos ${ }^{(2)(3)}$, \\ Gabriel Santos $^{(2)}$ \\ SiliconGate ${ }^{(2)}$, Lisboa, Portugal \\ marcelino.santos@silicon-gate.com \\ gabriel.santos@silicon-gate.com
}

\author{
Ângelo Monteiro ${ }^{(3)}$, \\ Alexandre Neves ${ }^{(3)}$, Pedro Braga ${ }^{(3)}$ \\ IST $^{(3)} /$ INESC-ID, Lisboa, Portugal \\ afrmonteiro@gmail.com,ahrn@sapo.pt, \\ pedro.r.braga@gmail.com
}

\begin{abstract}
Implementing monolithic DC-DC converters for low power portable applications with a standard low voltage CMOS technology leads to lower production costs and higher reliability. Moreover, it allows miniaturization by the integration of two units in the same die: the power management unit that regulates the supply voltage for the second unit, a dedicated signal processor, that performs the functions required. This paper presents original techniques that limit spikes in the internal supply voltage on a monolithic DC-DC converter, extending the use of the same technology for both units. These spikes are mainly caused by fast current variations in the path connecting the external power supply to the internal pads of the converter power block. This path includes two parasitic inductances inbuilt in bond wires and in package pins. Although these parasitic inductances present relative low values when compared with the typical external inductances of DC-DC converters, their effects can not be neglected when switching high currents at high switching frequency. The associated overvoltage frequently causes destruction, reliability problems and/or control malfunction. Different spike reduction techniques are presented and compared. The proposed techniques were used in the design of the gate driver of a DC-DC converter included in a power management unit implemented in a standard $0.35 \mu \mathrm{m}$ CMOS technology.
\end{abstract}

\section{INTRODUCTION}

$\mathrm{T}$ HE growing industry of portable equipment, in order to increase the level of integration, to reduce the equipment bill of materials and weight, integrates in the same die the power management unit (PMU) and the signal processor unit generally composed by analog and digital blocks, outlining a system-on-chip (SoC) [1]. The processor unit performs the functions required for the device application and the PMU regulates the supply voltage for the processor [2].

The integration of the PMU in a SoC using a standard CMOS low voltage technology can be a design challenge even using $I / O$ transistors (with higher voltage limits) if high currents and low cost packages (with bonding wires) are used.

In a PMU with a step-down DC-DC converter (buck), the components size of the off-chip LC filter should be small, in order to reduce print-circuit-board (PCB) area. However a reduced inductor value and a reduced capacitor value require a

This research work was partially funded by the Portuguese national project: PTDC/EEA-ELC/71412/06. high switching frequency in order to maintain successful energy transference from battery $\left(V_{B A T}\right)$ to the load $\left(v_{o u t}\right)$.

A step down DC-DC converter, operating at high switching frequency, produces fast current variations in the path connecting the external power supply source to the internal pads. When switching off the supply current, the fast current variation produces an internal supply voltage overshoot due to the parasitic inductances inbuilt in bond wires, $L_{B W}$, and in package pins, $L_{L F}$, on the serial path connected with the switches [3]. Although the low values of these parasitic inductances when compared with the external inductor of the buck converter, $L$, their effects can not be neglected when using high switching frequency and high current since they are the main reasons for spikes on internal supply voltage, $v_{I N}$. These spikes can cause overvoltage that leads to device destruction, reliability problems or malfunctions on the converter feedback control [1],[4-5].

These supply voltage spikes can be reduced in resonant converters. The class of resonant converters uses extra circuitry connected to the output load (load-resonant) or connected to the switches (switch-resonant) to force a sinusoidal shape on current and voltage in the switches. Although, the design of zero current switching (ZCS) or zero voltage switching (ZVS) resonant converters main objective is the reduction of switching loss, it can also reduce supply voltage spikes. However, the extra circuitry used in resonant converters (inductances and capacitors) are more affordable for discrete realizations [6]. For monolithic CMOS realizations a hard switching converter with an alternative solution, inbuilt in the gate driver of the power switches is presented in this paper.

In hard switching converters, supply voltages spikes can be reduced via controlled current variations through the parasitic inductances in the power supply path, with a moderate switch turn-off and turn-on time [1].

The techniques to reduce internal supply voltage spikes were used to design a gate driver for a buck converter fabricated with a $0.35 \mu \mathrm{m}$ CMOS process. The converter input voltage range $\left(V_{B A T}\right)$ is $1.8 \mathrm{~V}$ to $4.2 \mathrm{~V}$ and the output voltage $\left(v_{\text {out }}\right)$ is programmable in the range $1.2 \mathrm{~V}$ to $3.8 \mathrm{~V}$, with a maximum output load current of $180 \mathrm{~mA}$. The buck converter operates in discontinuous conduction mode (DCM) or in the 
frontier of continuous conduction mode (CCM). It is current controlled with variable switching frequency limited by $f_{s w} \leq 2 \mathrm{MHz}$; the controller limits the maximum current through the external inductance to $400 \mathrm{~mA}$.

In Section II, a conventional gate driver circuit is analyzed during the switching period. Section III presents a two slope gate driver circuit for supply voltage spikes reduction. Enhancements of two slope gate driver circuit are presented in Section IV, with a capacitive coupling scheme. Section V compares the previously presented solutions and, finally, Section VI presents the conclusions of this work.

\section{CONVENTIONAL GATE-DRIVER AND TRANSITOR SWITCHING BEHAVIORAL}

The simplest gate driver for the MOS power devices is a conventional buffer chain with appropriate transistor scaling [2],[7]. Fig. 1 shows this conventional gate driver, detailed for the $M_{p}$ power transistor (GDRVp), with the non-overlap subcircuit omitted for clearness. The body parasitic diode $D_{n}$ of power transistor $M_{n}$ is shown because the current $i_{L}$ flows through it when $M_{p}$ is turned-off, in order to preserve the inductor current, until the order to turn-on $M_{n}$ does not arrive from the controller (not shown). During this current switching, assuming that the external inductor current $\left(i_{L}\right)$ is nearly constant, the current decrease in $L_{p 1}$ parasitic inductor (HS - High Side path) is accomplish by a equal magnitude current increase in $L_{p 2}$ parasitic inductor (LS - Low Side path). If the value of $L_{p 1}$ and $L_{p 2}$ are equal then the voltage spike on the positive supply pad $\left(v_{I N}\right)$ is equal to voltage spike in the negative pad $(P G N D)$. The magnitude of this voltage spike $(\Delta v)$ is given by eq. 1 , assuming $L_{p}=L_{p 1}=L_{p 2}$ and $\Delta i_{1}=\Delta i_{2}=$ $\Delta i$; and $i_{1}+i_{2} \approx i_{L}$ and $i_{p}+i_{n}=i_{L}$.

$$
\Delta v=L_{p}|d i / d t|
$$

The above explanation states that the voltage spike on negative supply pad $(P G N D)$ is almost independent of $M_{n}$ turn-on time, but it is asserted by the $M_{p}$ turn-off time.

Theoretical electrical models for wire bonds and for device packages have been proposed in the literature with different level of accuracy depending on the application [3],[8-9]. In this work, a lumped resistance-inductor-capacitor $\left(R_{P} L_{P} C_{P}\right)$ parasitic model was adopted for wire bond and for package pins, but it will be represented by a parasitic inductor $\left(L_{p}=\right.$ $L_{B W}+L_{L F}$ ) for clearness (Fig.1).

In order to reduce supply voltage spikes $(\Delta v)$, some design alternatives to the gate driver circuit for $M_{p}$ switch are reported in the following sections, limiting the supply current slope. The gate driver circuit for $M_{n}$ switch is a conventional buffering scaling chain $(D R V n)$, and will be kept unchanged, when reporting the alternatives for $M_{p}$ gate driver.

\section{A. Conventional Fast Gate-Driver}

Two conflict criterions can be used to design a conventional gate driver (GDRVp) to the $M_{p}$ switch (Fig.1), with distinct results regarding turn-off time, maximum supply voltage spike, switching loss and silicon area.

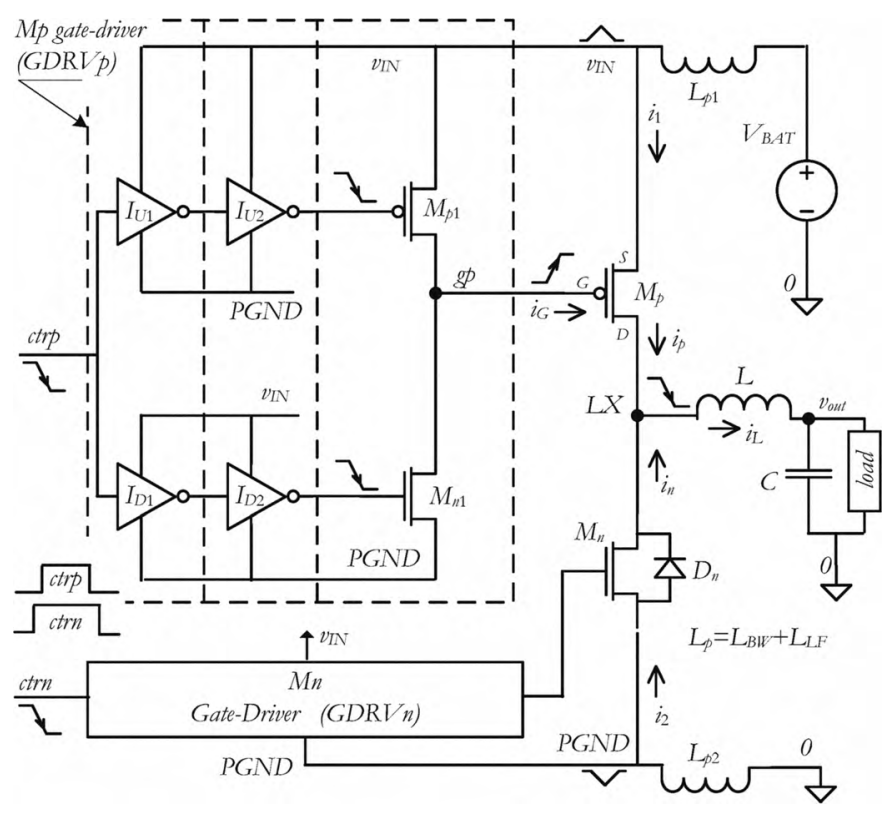

Fig. 1. Conventional gate driver circuit, detailed for $M_{p}$ transistor (GDRVp).

A conventional gate driver designed for fast turn-off and on times will generate low switching loss, but it requires a large silicon area. When switching off $400 \mathrm{~mA}$ with $8 \mathrm{~ns}$ turn-off time, the spike in $v_{I N}$ is approximately $0.8 \mathrm{~V}$, and the maximum $M_{p}$ source-drain voltage is $\left|v_{S D}\right|_{\max } \approx 6.3 \mathrm{~V}$ (with $V_{B A T}=4.2 \mathrm{~V}$ ).

\section{B. Conventional Slow Gate-Driver}

A conventional $M_{p}$ gate driver designed for reduced supply voltage spikes can be obtained by slowing down the turn-off and on times, but it will cause significant switching losses. The turn-off time for $M_{p}$ becomes $15 \mathrm{~ns}$ when designing the driver in order to limit the supply voltage spike to $0.5 \mathrm{~V}$ when $V_{B A T}=4.2 \mathrm{~V}$. The maximum $M_{p}$ source-drain voltage is $\left|v_{S D}\right|_{\max }$ $\approx 5.8 \mathrm{~V}\left(V_{B A T}=4.2 \mathrm{~V}\right)$.

The specifications of the step-down converter designed with a standard low-voltage CMOS technology, with a maximum switching frequency of $2 \mathrm{MHz}$, demanded a $M_{p}$ turn-off time of the conventional fast gate-driver (about 8ns), but it also demands a maximum supply voltage spike of the conventional slow gate driver (about $0.5 \mathrm{~V}$ for $V_{B A T}=4.2 \mathrm{~V}$ ), in order to limit the overvoltage across $M_{p}$ transistor. These two specifications are get-together simultaneously at expense of silicon area, to accommodate an enhanced two slope gate driver circuit.

In order to design a two slope gate driver circuit, the switching-off of the $p$-channel MOS transistor must be analyzed in detail.

\section{C. pMOS Switching-off Detail}

The simplified version of the $p$ MOS switching-off waveforms, composed only of piecewise linear sections and without scale, are presented in Fig.2. The following analysis assumes a null parasitic inductance $\left(L_{p}=0\right)$, in order to focus on main aspects of the $M_{p}$ transistor switching behavioral [1],[7],[10-11]. The analysis can be divided in four time frames: Before the first time frame $M_{p}$ transistor is fully conducting in the triode 
region, with a large overdrive, low $v_{S D p}$ voltage and the current through $M_{p}$ is increasing at an approximately linear rate given by $\left(v_{I N}-v_{o u t}\right) / L$, and $i_{1} \approx i_{p} \approx i_{L}$. At time frame one, when the gate voltage $\left(v_{G p}\right)$ of $M_{p}$ starts rising, the $v_{S G p}$ voltage starts to fall from $v_{I N}$ to a Miller plateau level. The voltage in the internal $M_{p}$ parasitic capacitors $C_{G S}$ and $C_{G D}$ is changing and the internal capacitors discharge-charge actions explains the difference observed at $M_{p}$ drain $\left(i_{p}\right)$ and source $\left(i_{1}\right)$ currents: part of $i_{p}$ cames from the charge of $C_{G D}$, but $i_{p} \approx i_{L}$. $M_{p}$ maintains the triode region with a decreasing overdrive, but all the current is flowing through it.

During time frame two, $M_{p}$ source-to-drain voltage $\left(v_{S D p}\right)$ goes from its initial value $\left(i_{1} R_{D S(o n)}\right)$ to the final value given by $v_{I N}+v_{D(o n)}$, exceeding the supply voltage. The source-to-gate voltage is kept constant at the Miller plateau level and the gate current charges the $C_{D S}$ and $C_{G D}$. During this time interval the $M_{p}$ drain current, $i_{p}$, is almost constant, since the transistor overdrive is also constant. The $M_{p}$ operation point moves from triode to saturation at the same time as the source-to-drain voltage raises.

On the third time frame, the $M_{p}$ drain current, $i_{p}$ falls from the predefined value toward zero at the end of interval, while the source-to-gate voltage $\left(v_{S G P}\right)$ decreases from the Miller plateau level to the $M_{p}$ threshold level $\left|V_{T p}\right|$. During this time interval $M_{p}$ transistor is operating in saturation but it is shifting to the cut-off region. The gate current, $i_{G}$, is chargingdischarging the internal $M_{p}$ capacitors $C_{G D}$ and $C_{G S}$. In order to ensure the continuity of $i_{L}$ current, that is almost constant during this time frame, the $M_{n}$ body diode turns-on automatically, compensating the variation of $i_{p}$ with identical variation at $i_{2}$, that flows through $D_{n}$ while $M_{n}$ is off. As soon as this parasitic diode turns-on, the voltage at $L X$ node $\left(v_{L X}\right)$ drops from its initial value $\left(v_{I N}-i_{1} R_{D S(o n)}\right)$ to a clamped negative voltage defined by the forward bias diode.

During time frame four the $M_{p}$ source-to-gate voltage drops form $\left|V_{T p}\right|$ to zero, at the same time as the gate current $\left(i_{G}\right)$ full charges-discharges $C_{G D}$ and $C_{G S}$. At this time interval the drain current $\left(i_{p}\right)$ is zero. The external main inductance current flows through the parasitic diode, $D_{n}$, setting $i_{L}=i_{n}=i_{2}$. These currents are now decreasing at a rate given by $\left(v_{\text {out }}+v_{D(o n)}\right) / L$. In practice, this time interval imposes a dead-time delay that avoids $M_{p}$ and $M_{n}$ simultaneous conduction, which would cause an undesired shoot-through current.

After time frame four, the DC-DC controller maintains $M_{p}$ cut-off and it turns-on the synchronous rectifier transistor $\left(M_{n}\right)$, in order to reduce power losses from $i_{2} v_{D(o n)}$ to $\left.i_{2} \mid v_{D S h(o n)}\right)$.

The last signal waveform at Fig.2 $\left(\left|v_{S D p} \cdot i_{p}\right|\right)$ represents the switching power losses during $M_{p}$ turn-off sequence. These power losses depend on the duration of time frames two and three. The duration of all time intervals depends on tree main factors: $M_{p}$ parasitic capacitors; $M_{p}$ gate-driver strength; and $M_{p}$ threshold and overdrive voltages.

The four time frames at Fig.2 have solid straight lines frontier definition, but HSpice waveform simulations show more flexibility to define the time frames border lines. For example, the current through $M_{p}$ transistor $\left(i_{1}, i_{p}\right)$ starts to
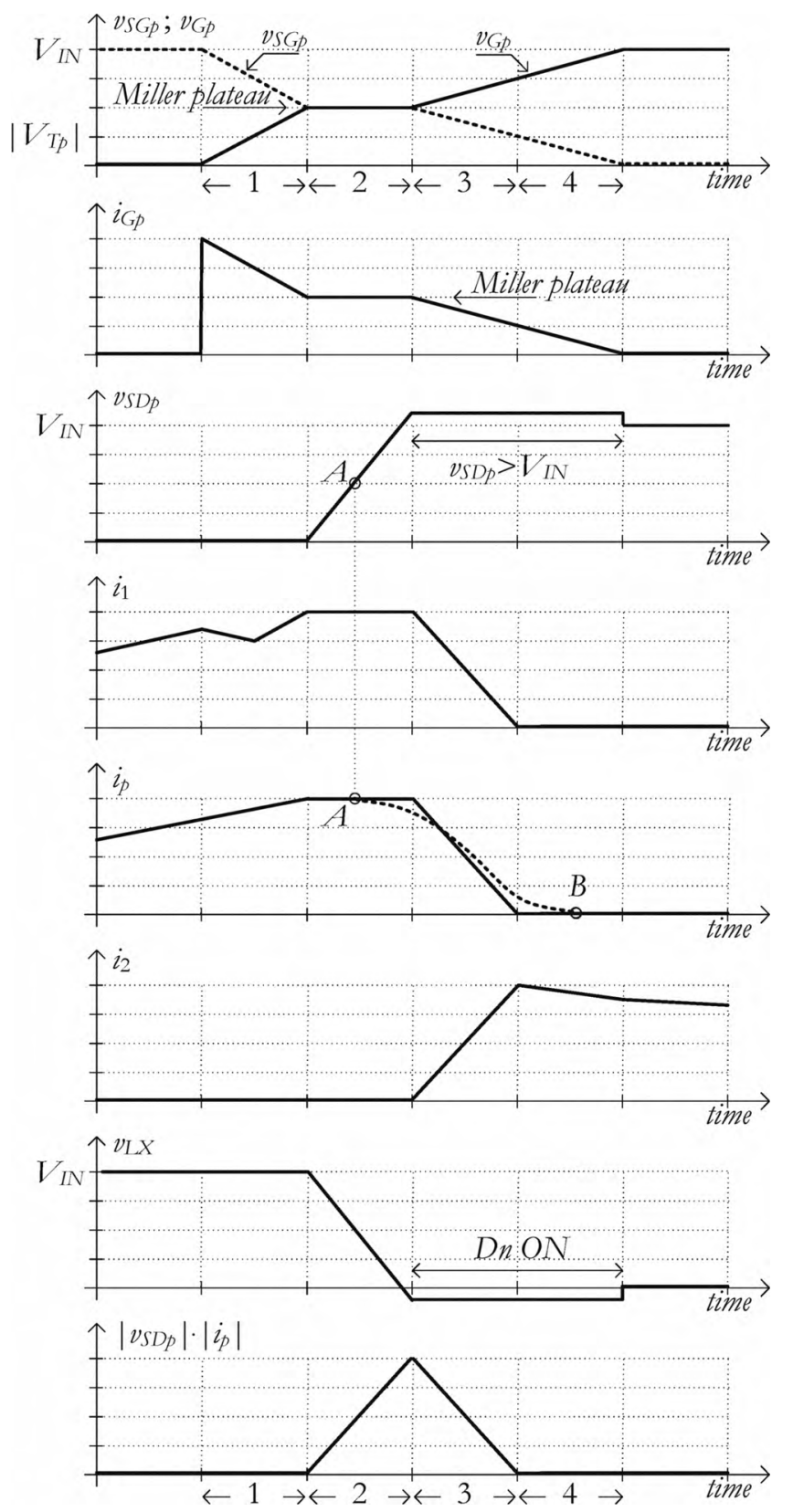

Fig. 2. Simplified waveforms when $M_{p}$ switch (high side) turns-off.

decrease at time frame two and ends at time frame four, which is point up by $A-B$ dashed line waveform at Fig.2.

The $A-B$ dashed line shows a more realistic current waveform through $M_{p}$ in order to define the $M_{p}$ cut-off time criterion: the time from $v_{S D P}=1 / 2 \cdot V_{B A T}$ (point- $A$ ) to null drain current (point- $B$ ).

The simplified waveforms at Fig.2 were sketched assuming zero parasitic inductance in the bonding wires and package pins $\left(L_{p}=0\right)$. Nevertheless, Fig. 2 shows that the major influence of those parasitic inductances occurs at time frame three, where the current through $M_{p}$ falls to zero and the current through the $L S$ path $\left(M_{n}, D_{n}\right)$ increases with identical rate, causing supply voltage spikes in $v_{I N}$ and $P G N D$. Some influence of the parasitic inductances will also be noticeable during time frame one, when the charge-discharge of $C_{G S}$ and $C_{G D}$ cause variations on $i_{1}$. 


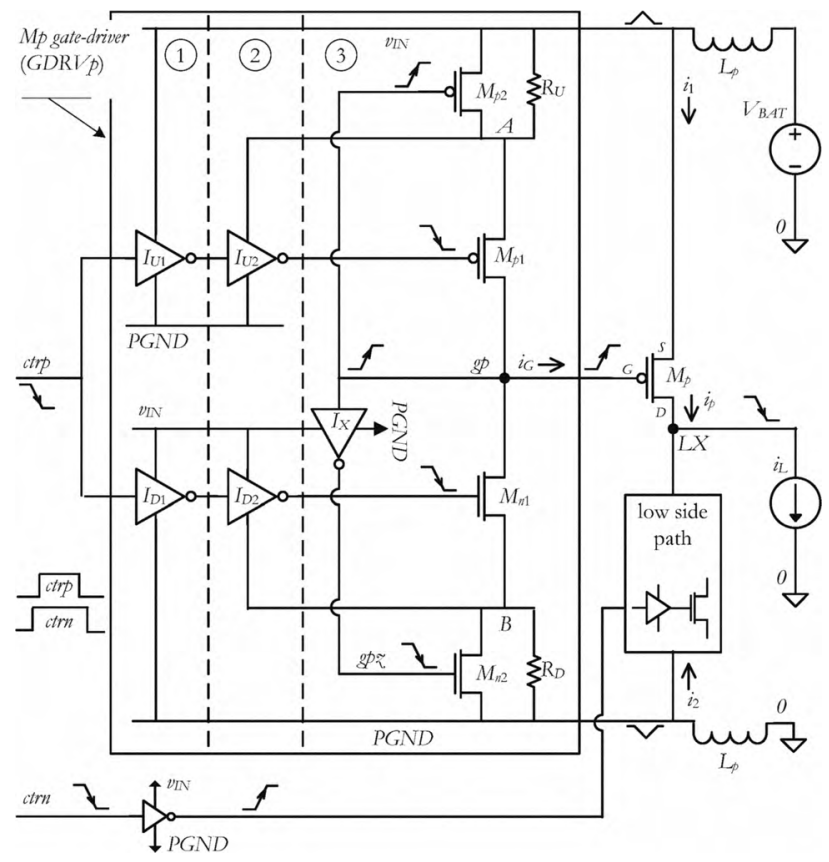

Fig. 3. Simple two slope gate driver circuit (GDRVp).

In order to reduce the voltage spikes in $v_{I N}$, and $P G N D$ the $M_{p}$ gate driver must control the current slope through the parasitic inductances.

\section{TWO SLOPE GATE DRIVER}

The main propose of this two slope gate-driver is to reduce time intervals one and two (Fig.2), and to expand time interval three. Fig. 3 shows the simple two slope gate-driver circuit $(G D R V p)$ for $M_{p}$. It also shows a block ( $L S$ path) representing the $M_{n}$ MOS switch and the respective conventional gatedriver circuit (GDRVn, $\left.M_{n}, D_{n}\right)$.

The simple two slope circuit is composed by the three sections marked in Fig. 3. Section one and two are identical to the conventional gate-driver circuit, with a tapering buffer chain. Section three, the last driver stage, charges or discharges the $M_{p}$ parasitic gate capacitors with two different current strengths.

The two slope driver uses a resistor $\left(R_{U}\right)$ in series with the pull-up path that turns off $M_{p}$ in order to retard time frame three. This resistor is bypassed by an additional $p \mathrm{MOS}, M_{p 2}$, which allows the reduction of time frames one and two. Since, for the intended operation, $M_{p 2}$ must be on when $M_{p}$ is on (shrinking time frame one and two) and it must be off when $M_{p}$ is sifting to off (delaying time frame three), the gate of $M_{p 2}$ can be controlled by the same signal as the gate of $M_{p}$. Ideally $M_{p 2}$ should be completely off when $M_{p}$ is moving from saturation region to off region (time frame three).

The same approach is illustrated in Fig. $3\left(M_{n 2}\right.$ and $\left.R_{D}\right)$ for the control of $M_{p}$ turn-on time.

\section{ENHANCED TWO SLOPE GATE DRIVER}

Lower voltage spikes with maximum supply voltage $\left(V_{B A T}\right)$ can be obtained using capacitor coupling techniques superimposed to the two slope gate driver circuit. Fig. 4 shows

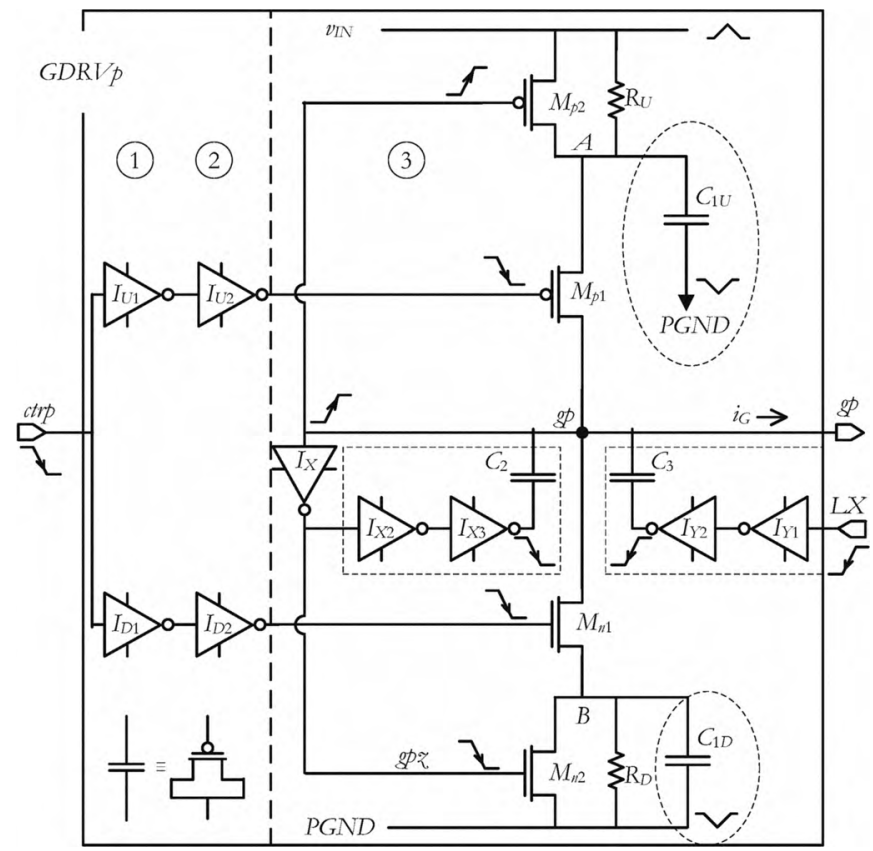

Fig. 4. Enhanced two slope gate driver circuit (all-in-one coupling).

three different useful capacitor couplings. Each of these solutions can be used independently and their effects will be explained separately.

Fig.4 shows the gate-driver of Fig.3 (GDRVp), with additional sub-circuits in section three. The added sub-circuits are inside the dashed lines and they only modify the gatedriver behavior during the switching transitions.

\section{A. PGND coupling $\left(C_{1}\right)$}

The sub-circuit inside the two dashed oval lines consists only in two capacitors $\left(C_{1 U}, C_{1 D}\right)$ implemented by MOS transistors, coupling the internal $P G N D$ supply to nodes $A$ and $B$.

This sub-circuit works as follows: During a Low to High (L-H) transition at node $g p$ ( $M_{p}$ gate) the source-drain current through $M_{p}$ starts to fall, and the supply pads parasitic inductances $\left(L_{p}\right)$ produce an overshoot in $v_{I N}$ and an undershoot in $P G N D$. This voltage decrease is coupled to node $A$ through $C_{1 U}$ capacitor, reducing $M_{p 1}$ overdrive and therefore reducing the driver strength and smoothing the current variation in $M_{p}$.

\section{B. gp delay coupling $\left(C_{2}\right)$}

An alternative way to reduce voltage spikes using capacitive coupling is implemented adding inverters $I_{X 2}, I_{X 3}$ and the capacitor $C_{2}$ (Fig.4).

When the node $g p$ is at low state, the $M_{p}$ switch is closed, the output of $I_{X 3}$ inverter is at high state and $C_{2}$ capacitor is charged. The $M_{p}$ switch opening process begins by raising the $g p$ node voltage from zero toward $v_{I N}$. The $g p$ node voltage increases by charging-discharging all parasitic capacitors connected to it, and also by discharging $C_{2}$ capacitor.

After a rising edge of node $g p$ (with a delay defined by $I_{X 2}$ and $I_{X 3}$ propagation delays), the output of $I_{X 3}$ perform a fast $\mathrm{H}$ $\mathrm{L}$ transition, slowing down the voltage increase at $g p$ node. Ideally, this transition occurs when $M_{p}$ source-to-gate voltage 


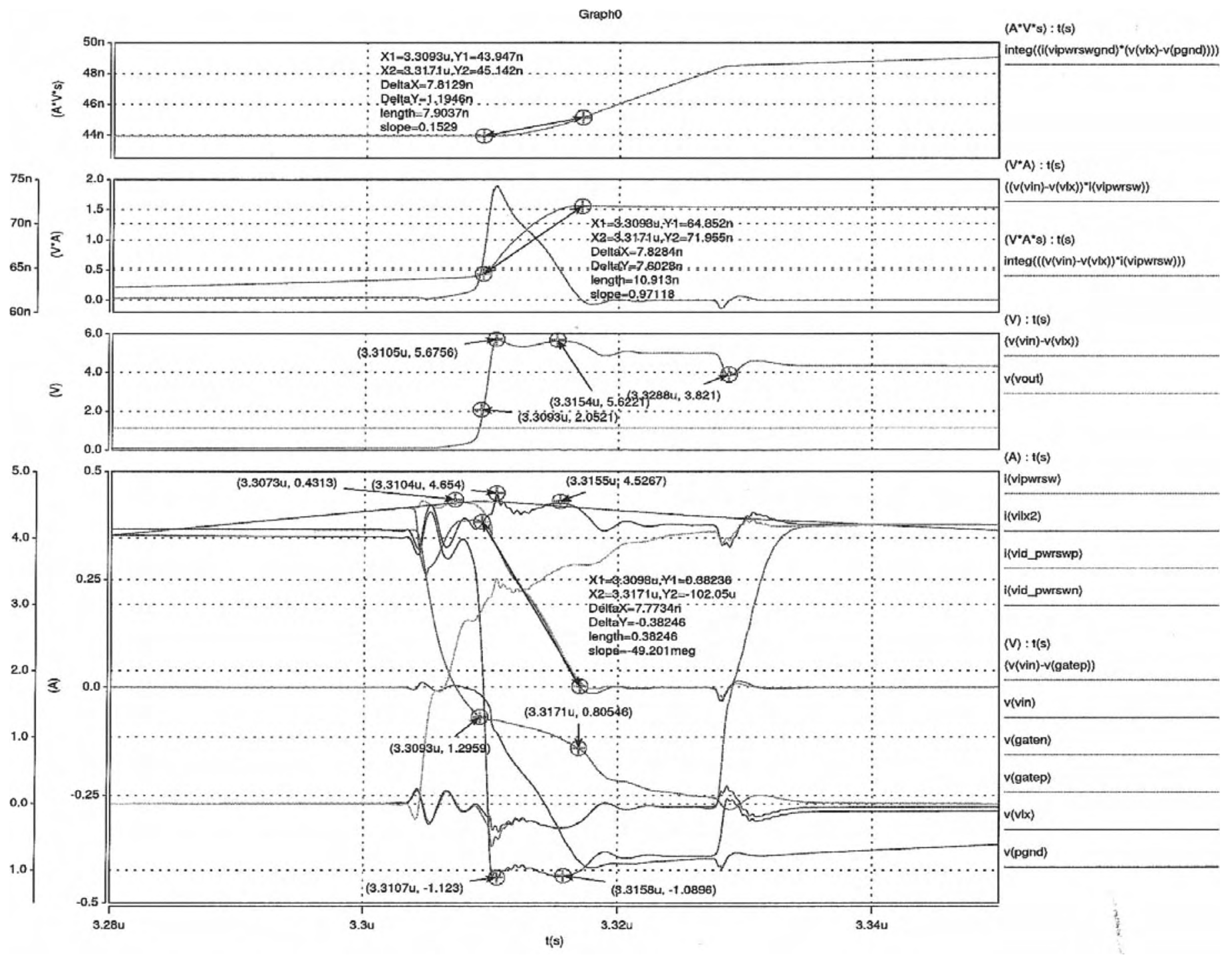

Fig. 5. Enhanced two slope gate driver signals waveforms ( $g p$ delay coupling).

is decreasing from Miller plateau level to the threshold level (Fig.2), reducing $M_{p}$ high current slope and therefore the undesired voltage spikes.

The same sub-circuit produces a coupling scheme during the $M_{p}$ turn-on process. In this case the $g p$ voltage decreasing rate is slowed down when the output of $I_{X 3}$ inverter performs an L-H transition.

The coupling strength introduced by $C_{2}$ can be different at the $M_{p}$ turn-on and off. This difference can be achieved by designing the inverter $I_{X 3} \mathrm{H}-\mathrm{L}$ transition time different from the L-H transition time.

Fig.5 shows the simulation waveforms when $I_{X 3}$ inverter outputs a faster H-L transition. Other sub-circuits in dashed boxes of Fig. 4 were dismissed for this gate-driver simulation. The maximum supply voltage spike when turning off $400 \mathrm{~mA}$ is $0.5 \mathrm{~V}\left(V_{B A T}=4.2 \mathrm{~V}\right)$, and the turn-off time for $M_{p}$ switch is $8 \mathrm{~ns}$. The maximum $M_{p}$ source-drain voltage is $\left|v_{S D \mathrm{p}}\right|_{\max } \approx 5.7 \mathrm{~V}$.

\section{LX feedback coupling $\left(C_{3}\right)$}

Alternatively, the reduction of supply voltage spikes by capacitive coupling can be achieved by adding the inverters
$I_{Y 1}$ and $I_{Y 2}$ and capacitor $C_{3}$ (Fig.4). The inverter $I_{Y 1}$ input is node $L X$ (Fig.1).

The sensing in this coupling scheme takes advantage of the fact that a fast current decrease on $M_{p}$ switch is automatically compensated by a symmetrical current increase on the low side path in order to maintain the external current $\left(i_{L}\right)$. Fig.2 waveforms show that the $L X$ node voltage decreases quickly from $v_{I N}$ to a negative value as soon as the $M_{p}$ drain current begins to decrease. This $\mathrm{H}-\mathrm{L}$ voltage variation at $L X$ node propagates through $I_{Y 1}$ and $I_{Y 2}$ and is applied to $C_{3}$. A fast H-L transition at $I_{Y 2}$ output slows down the increase rate at $g p$ node. Similarly to the $g p$ delay coupling described before, the $L X$ feedback coupling scheme also works during the $M_{p}$ turn-on process.

The similarity of $L X$ feedback coupling and $g p$ delay coupling schemes is obvious. However, the $L X$ feedback coupling scheme, as described, can lead to circuit instability. When both power switches are off, the voltage in node $L X$ has two components: 1) an average DC value of $v_{\text {out }}$ and 2) a sinusoidal oscillation with decaying amplitude. The second component is the underdamped response of the external inductor in series with the high resistance of the open switches 
$\left(R_{D S(o f f)}\right)$ and the parasitic capacitors in node $L X$. The oscillation at $I_{Y 2}$ output, propagated through $C_{3}$ to $M_{p}$ and to the $L X$ node, introduces positive feedback that can maintain the oscillation.

\section{RESULTS}

Table-I shows measurements from the conventional slow and fast gate-drivers and also from the two slope gate-driver circuit with three different alternatives: simple; with $P G N D$ coupling and with $g p$ delay coupling. $|\Delta v|_{\max }$ is the maximum supply spike magnitude in $P G N D$ node or on the $v_{I N}$ node. $\left|v_{S D p}\right|_{\max }$ is the maximum source-to-drain voltage at $M_{p} . t_{o f f}$ is the $M_{p}$ cut-off time as previously defined. $t_{S N S 0}$ is the delay time from when $M_{p}$ gate voltage starts to rise until $i_{p}=0$. rate 1 is the ratio of two areas, $(W \cdot L)_{G D R V p}$ and $(W \cdot L)_{M p}$; where $(W \cdot L)_{M p}$ is the $M_{p}$ transistor width multiplied to its length and $(W \cdot L)_{G D R V p}$ is the sum of all transistors areas (excluding MOS capacitors and $I_{Y \mathrm{n}}$ and $I_{X \mathrm{n}}$ inverters) in the gate-driver circuit. rate2 is the ratio of two areas, $(W \cdot L)_{M O S C a p}$ and $(W \cdot L)_{M p}$; where $(W L)_{M O S C a p}$ is the sum of capacitors $\left(C_{u 1}\right.$ or $\left.C_{2}\right)$ and inverters areas $\left(I_{X \mathrm{n}}\right.$ for $\left.C_{2}\right) . W_{S W P L}$ is the $M_{p}$ turn-off energy loss. This energy loss results from time integration of the product $\left|v_{S D p}\right| .\left|i_{p}\right|$ during the $M_{p}$ turn-off $\left(t_{o f f}\right)$ time. $W_{D n L}$ is the energy loss of the low side transistor body diode $\left(D_{n}\right)$ during $M_{p}$ turnoff time interval. This energy loss is a slice of the total body diode loss, since the integration interval excludes the nonoverlap switching dead-time.

Table-I shows that a significant reduction on the maximum supply voltage spike can be achieved at the expense of gatedriver silicon area. The enhanced two slope gate-driver circuit with $g p$ delay coupling presents low voltage spikes $\left(|\Delta v|_{\max }\right)$ as the conventional slow gate-driver and a $M_{p}$ turn-off time $\left(t_{o f f}\right)$ identical to the conventional fast gate-driver. The superior performance of the two slope enhanced gate-driver costs a significant silicon area overhead when compared to the conventional gate-driver. Although the area overhead, the enhanced two slope gate-driver circuits is a suitable solution when a low voltage CMOS technology is used to design high switching frequency, high current, DC-DC step-down

Table-1. Gate-driver circuit performance measured parameters $\left(V_{B A T}=4.2 \mathrm{~V}, v_{\text {out }}=1.2 \mathrm{~V},\left(I_{L}\right)_{\max }=0.4 \mathrm{~A}\right)$.

\begin{tabular}{|c|c|c|c|c|c|}
\hline \multirow{2}{*}{$\begin{array}{c}\text { GDRV type } \rightarrow \\
\text { parameter [unit] } \\
\downarrow\end{array}$} & \multicolumn{2}{|c|}{ conventional } & \multicolumn{3}{|c|}{ two slope } \\
\hline & slow & fast & simple & $\begin{array}{c}\text { PGND } \\
\text { coupling }\end{array}$ & $\begin{array}{c}g p \\
\text { coupling }\end{array}$ \\
\hline$|\Delta v|_{\text {MAX }}[\mathrm{V}]$ & 0.5 & 0.7 & 1.0 & 0.7 & 0.5 \\
\hline$\left|V_{D S p}\right|_{\text {MAX }}[\mathrm{V}]$ & 5.7 & 6.3 & 6.9 & 6.3 & 5.7 \\
\hline $\boldsymbol{t}_{\text {off }} \quad[\mathrm{ns}]$ & 14.8 & 7.9 & 3.2 & 4.4 & 7.8 \\
\hline$T_{S N S O}$ & 60 & 40 & 9 & 10 & 16 \\
\hline$W_{S W P L}$ & 14.8 & 8.8 & 3.8 & 5.0 & 7.6 \\
\hline$W_{D n L} \quad[\mathrm{~nJ}]$ & 3.2 & 1.4 & 0.4 & 0.6 & 1.2 \\
\hline rate1 $[\%]$ & 0.3 & 0.4 & 14 & 14 & 14 \\
\hline rate2 $[\%]$ & 0 & 0 & 0 & 17 & 23 \\
\hline
\end{tabular}

converters.

The gate-driver with $P G N D$ coupling, for a similar voltage spike value, is able to turn-off $M_{p}$ much faster and with lower losses. $P G N D$ coupling is less sensible to battery voltage $\left(V_{B A T}\right)$, which can be an advantage over the $g p$ delay coupling.

\section{CONCLUSIONS}

The design space of a gate-driver circuit for the HS MOS switch was addressed, with the objective to minimize supply voltages spikes due to the parasitic bonding wire and package pins inductances.

An original gate-driver circuit topology labeled two-slope gate-driver was proposed with three capacitor coupling optimizations. The proposed gate drives, for similar supply voltage spikes, presents faster turn-off times and significantly lower losses. Although the proposed drivers exhibit higher area overhead than conventional drivers, their use can be a suitable solution when low voltage CMOS technologies are used to design high current, high frequency, DC-DC converters.

$L X$ capacitive coupling is the solution less sensitive to the operating conditions $\left(V_{B A T}, v_{\text {out }}\right)$ but can lead to an unstable behavior. This solution will be addressed in future works.

\section{REFERENCES}

[1] H. Deng, A.Q. Huang and Y. Ma, "Design of a monolithic high frequency fast transient buck for portable application," 35th Annual IEEE Power Electronics Specialists Conference, pp. 4448-4452, 2004.

[2] V. Kursun, S.G. Narendra, V.K. De and E.G. Friedman, "High Input Voltage Step-Down DC-DC Converters for Integration in a Low Voltage CMOS Process," 5th International Symposium on Quality Electronic Design, pp. 517-521, September 2004.

[3] Lawrence Larson and D. Jessie, "Advances in RF Packaging Technologies for Next-Generation Wireless Communications Applications (Invited Paper)," IEEE Custom Integrated Circuits Conference, pp. 323-330, 2003.

[4] V. Kursun, S.G. Narendra, V.K. De and E.G. Friedman, "Cascode monolithic DC-DC converter for reliable operation at high input voltages," Analog Integr. Circuits Signal Process, vol. 42, no. 3, pp. 231-238, Mar. 2005.

[5] J.F. Rocha, M.B. Santos, J.M.D. Costa and F.A. Lima, "Level Shifters and DCVSL for a Low-Voltage CMOS 4.2-V Buck Converter," IEEE Transactions on Industrial Electronics, vol. 55, no. 9, pp. 3315-3323, September 2008.

[6] Z. Zhang, W. Eberle, Z. Yang, Y.F. Liu and P.C. Sen, "Optimal Design of Resonant Gate Driver for Buck Converter Based on a New Analytical Loss Model," IEEE Transactions on Power Electronics, vol. 23, no. 2, pp. 653-666, March 2008.

[7] L. Balogh, "Design and Application Guide for High Speed MOSFET Gate Drive Circuits," TI Power Design Seminar, pp. 1-37, 2001.

[8] F. Alimenti, P. Mezzanotte, L. Roselli and R. Sorrentino, "Modeling and Characterization of the Bonding-Wire Interconnection," IEEE Transactions on Microwave Theory and Techniques, vol. 49, no. 1, pp. 142-150, January 2001.

[9] Alan Elbanhawy, "Are Traditional MOSFET Packages Suitable For the New Generation of DC-DC Converters," 4th International Power Electronics and Motion Control Conference, pp. 316-319, 2004.

[10] Alan Elbanhawy, "Mathematical Treatment for HS MOSFET Turn Off," 5th International Conference on Power Electronics and Drive Systems (PEDS), pp. 81-84, 2003.

[11] J. Brown, "Modeling the Switching Performance of a MOSFET in the High Side of a Non-Isolated Buck Converter," IEEE Transactions on Power Electronics, vol. 21, no. 1, pp. 3-10, January 2006. 